\title{
A Post-Contingency Power Flow Emulator for Generalized Probabilistic Risks Assessment of Power Grids
}

\author{
R. Rocchetta \& E. Patelli \\ Institute for Risk and Uncertainty, Liverpool University \\ Liverpool, United Kingdom \\ Chadwick Building, Liverpool L69 7ZF \\ roberto.rocchetta@liverpool.ac.uk \\ edoardo.patelli@liverpool.ac.uk
}

\begin{abstract}
In the past decades, risk assessment frameworks have been developed as a viable alternative to Security-Constrained Dispatch for power grids. Risk-based dispatch can increase power grid profitability and help better understanding of prominent hazards, however, relevant sources of uncertainty are often neglected to meet efficiency requirements. In this work, a research effort towards the development of advanced risk assessment frameworks for power grids is presented. A surrogate model strategy is proposed and speeds up the post-contingency consequence evaluation by analytical sensitivity indices (Line-Outage Distribution Factor). The risk surrogate model is embedded within a generalized uncertainty quantification framework to evaluate: (1) The amount of epistemic uncertainty affecting the risk index and attributable to imprecision in the parameters of the model; (2) The effect aleatory uncertainty in environmental-operational variables. The surrogate model is tested on the 24 nodes IEEE Reliability Test System. Computational time and accuracy of the results are compared to a high-fidelity AC power flow risk model. Strengths and weaknesses of the proposed framework highlighted.
\end{abstract}

Keywords: Power Grid, Risk Assessment, Line-Outage-Distribution-Factors, Surrogate, Probability Boxes, Sensitivity Analysis

\section{Introduction}

The goal of a power grid operator is to satisfy the customer's electric needs in the safest and most economical way. To guarantee high profitability and operational safety, the grid operator will schedule an optimal generation profile which minimizes overall production costs and, at the same time, fulfill a set of security-related constraints [1]. For instance, the scheduled production will have to comply with the thermal limit of the transmission lines, with the maximum capacity of the generators and prove to be safe and stable for a predefined list of contingencies (e.g. a set of N-k failures). This optimization procedure is known as Security Constrained Optimal Power Flow (SCOPF) or Security Constrained Unit-Commitment, see e.g. [2, 3, 4] or [5]. The power generation profile obtained from the SCOPF will be safe even after the occurrence of one of the listed failures. 
SCOPF is a trusted approach, however, it can lead to non-optimal profits, penalizing the overall economic gain of the network due to its hard constraints on the gird safety. Power grid safety and revenues are in fact conflicting objectives [6] ard hard security constraints can not exploit the existing risk-cost trade-off [7]. To overcome this limitation, online risk assessment frameworks have been developed, providing a viable alternative to security constrained dispatch, see e.g. $[6,8,9,10]$. Compared to a SCOPF solution, risk-informed dispatch can lead to a comparable performance in terms of risks but with higher expected profits. This is due to the enhanced awareness of relevant operational jeopardies [7, 10]. Risk assessment frameworks are powerful and versatile tools, although a few limitations have to be addressed. To meet efficiency requirements simplifying assumptions are generally introduced and there is a tendency to disregard relevant sources of uncertainty which can substantially affect expected risk and profit of the system (e.g. variability in weather factors, load demand, components availability, etc.).

In this work, we present a computationally efficient risk assessment framework which accounts for relevant sources of uncertainty. A surrogate model strategy, based on linear approximations of sensitivity indices known as Line Outage Distribution Factors (LODF), is used to reduce the computational cost of each risk evaluations. The surrogate model is embedded within a generalized Uncertainty Quantification (UQ) framework and tested in performing various UQ tasks [11]. Mixed sources of uncertainty are taken into account within the framework and their separate effect quantified in the risk score. Specifically, aleatory uncertainty affecting future weather conditions and power demand and imprecision (i.e. the epistemic uncertainty) affecting the probabilistic parameters of the risk model are accounted for. Probability boxes (P-boxes) are a powerful and versatile tool and are used within the framework to model factors affected by mixed sources of uncertainty, see e.g. $[12,13,14,15,16,17,11]$. Uncertainty propagation and sensitivity analysis quantify the effect of imprecision and inherent variability on the risk index. The framework is tested on the 24 nodes IEEE Reliability Test System. Strengths and weaknesses of the proposed surrogate model are discussed in terms of its accuracy and efficiency when compared with a high-fidelity risk model based on AC optimal power flow solver. Classical UQ tasks, i.e. Monte Carlo propagation, and generalized UQ tasks, i.e. P-boxes slicing propagation and sensitivity analysis, are both performed.

The rest of this paper is organized as follows: Section 2 introduces the generalized UQ framework, P-box characterization, propagation, and sensitivity analysis. The severity model and weather-induced failure morel are presented in Section 3. The proposed surrogate risk model is presented in Section 4 and is applied to a real-world power grid case study in Section 5 and results are discussed in Sections 6. Section 7 closes the paper.

\section{A generalized framework for uncertainty quantification}

Aleatory and epistemic are two different types of uncertainties, sometimes referred to as inherent variability and lack of knowledge, respectively $[17,18]$. The epistemic uncertainty can be reduced by further data collection, at least in principle. From an intuitive standpoint, input/output of analysis for which information is abundant and high in quality will be affected by a mild or negligible effect of epistemic uncertainty (although this is generally difficult to judge a priori [13]). In those cases, pure probabilistic methods will be suitable to perform uncertainty quantification tasks (e.g. the uncertainty characterization handled by joint PDF, CDF and/or random 


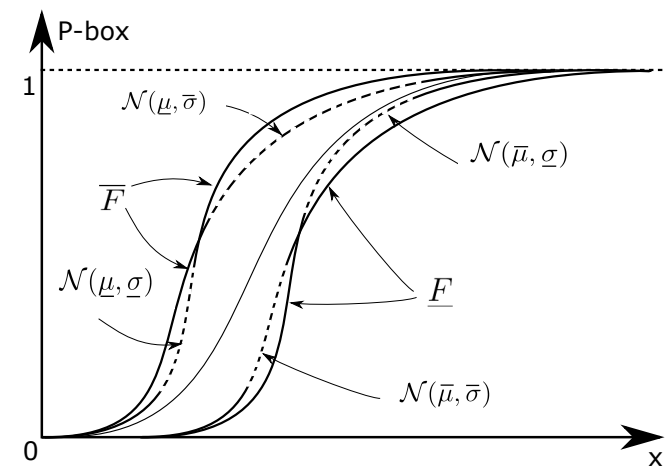

Figure 1: An example of distribution P-box with Gaussian distribution family and imprecise mean and standard deviation.

processes and the propagation of the uncertainty carried out with plain Monte Carlo).

On the other hand, in situations for which data is not abundant, the background knowledge limited and inherently variable factors are affecting the analysis, both aleatory and epistemic uncertainties will have a relevant role. In those cases, the use of pure probabilistic approaches may lead to an underestimation of the uncertainty [13]. The underestimation is attributable to modeling assumptions needed to characterize the imprecision in a probabilistic framework (e.g. assume a distribution family or assume a crisp estimation of the distribution's parameters given just a few samples). To overcome those limitations, traditional UQ frameworks have been extended to generalized UQ frameworks, which are capable of handling mixed sources of uncertainty and with less or no need for artificial model assumptions. Some examples of most widely applied mathematical tools to model quantities affected by mixed aleatory epistemic uncertainties are: Dempster-Shafer structures (DSS) [19], Fuzzy variables [17], info-gaps [20] and probability boxes (P-Boxes) [12].

\subsection{Probability boxes}

Probability Boxes (P-boxes) are powerful mathematical tools to characterize factors affected by mixed sources of uncertainty. P-boxes have grown in popularity thanks to their versatility and powerful uncertainty quantification capability $[12,21]$. and are used in this work to model factors affected by mixed aleatory-epistemic uncertainties. P-boxes have been recently applied for reliability analysis [15], [16], [17], in the context of evidential networks [22], and also for power grid analysis [13], [14].

A P-box is defined as a pair of CDFs bounding the probability between upper and lower bounds. Depending on the available background information, the underlying distribution family can be defined (i.e. distributional/parametric P-box) or only the bounds on the CDF can be available (i.e. distribution-free P-boxes). An example of Gaussian parametric P-box for which mean $\mu$ and standard deviation $\sigma$ are imprecisely defined is presented in Fig. 1. P-boxes can be constructed from data and for further details on the subject the reader is reminded to [12].

Before defining mathematically a P-box, let first recall the definition of cumulative distribution functions (CDFs), which is a non decreasing mapping from $\mathbb{R}$ to $[0,1]$ such that for a probability measure $P$ and for each $p \in \mathbb{R}$, the following $F_{P}(p)=P((-\infty, p])$ holds. P-boxes is 
a pair of $\operatorname{CDFs}[\underline{F}, \bar{F}]$ such that $\underline{F}$ dominates $\bar{F}$ in a probabilistic sense. A P-box can be viewed as a continuous form of random set and defined as follows:

$$
\left\{P \in \mathbb{P}_{\mathbb{R}} \mid \forall x \in \mathbb{R}, \underline{F}(x)=P([-\infty, x]) \leq \bar{F}(x)\right\}
$$

where equation (1) defines the credal set induced by the P-box $[\underline{F}, \bar{F}]$.

\subsection{Uncertainty propagation for P-boxes}

Uncertainty characterized using P-boxes can be propagated to the output of a computational model $y=\mathcal{R}(\mathbf{x})$, for instance, a risk assessment model in this work. For the propagation, several methods can be used: double loop MC [23], importance sampling method [24], slicing method [13], multi-level meta-models [16] and, for special classes of problems, analytical methods [25]. In this work, the slicing method is used to propagate P-box uncertainty on the line of previous works [11].

\subsubsection{The slicing method}

In slicing method (sometime named focal element propagation method) a total of $N_{s}$ independent 'alpha-cuts' are directly obtained from the P-box bounds. An ' $\alpha$-cut', or ' $\alpha$-slice', is obtained by sampling a value $\alpha$ from a uniform probability distribution $U(0,1)$ and inverting lower and upper bounds of the P-box as follows:

$$
\begin{aligned}
& \bar{F}_{X, i}(\alpha)^{-1}=\left\{x \mid \bar{F}_{X, i}(x)=\alpha\right\}, \alpha \in[0,1] \\
& \underline{F}_{X, i}(\alpha)^{-1}=\left\{x \mid \underline{F}_{X, i}(x)=\alpha\right\}, \alpha \in[0,1]
\end{aligned}
$$

The interval $\left[\bar{F}_{X, i}(\alpha)^{-1}, \underline{F}_{X, i}(\alpha)^{-1}\right]$ is the support of the focal element for the $i^{\text {th }}$ input P-box. Cartesian product of the input focal elements defines a sample hyper-rectangle (i.e. n-orthotope):

$$
\mathcal{I}_{X, \alpha}:\left[\underline{F}_{X 1}\left(\alpha_{1}\right)^{-1}, \bar{F}_{X 1}\left(\alpha_{1}\right)^{-1}\right] \times \ldots \times\left[\underline{F}_{X n}\left(\alpha_{n}\right)^{-1}, \bar{F}_{X n}\left(\alpha_{n}\right)^{-1}\right]
$$

Once a sample support $\mathcal{I}_{X, \alpha}$ is obtained, the output bounds can be computed by solving an optimization problem constrained in $\mathcal{I}_{X, \alpha}$ :

$$
\begin{aligned}
& \bar{y}_{i}=\max _{\mathbf{x} \in I_{X, \alpha}} \mathcal{R}(\mathbf{x}) \\
& \underline{y}_{i}=\min _{\mathbf{x} \in I_{X, \alpha}} \mathcal{R}(\mathbf{x})
\end{aligned}
$$

where $\bar{y}_{i}$ and $\underline{y}_{i}$ are the maximum and minimum output responses associated to the $\alpha^{\text {th }}$ slice, respectively.

Slices are sampled until a predefined number $N_{\alpha}$ of hyper-rectangles are samples. Then, upper and lower $\mathrm{CDF}$ bounds for the output response of interest are be computed as follows:

$$
\underline{F}_{Y}(y)=\frac{1}{N_{s}} \sum_{i=1}^{N s} 1_{y \leq \bar{y}_{i}} \quad \bar{F}_{Y}(y)=\frac{1}{N_{s}} \sum_{i=1}^{N s} 1_{y \leq \underline{y}_{i}}
$$

To calculate $\bar{y}_{i}$ and $y_{i}$, different strategies can be adopted, for instance, the sampling method [13], the vertex method [26], the interval arithmetic method [27] or a global optimization strategy [28]. 


\subsection{Sensitivity Analysis for P-boxes}

Sensitivity analysis is generally used to investigate the importance of model inputs for an output of interest. Variance-based sensitivity measures, e.g. Sobol's indices, have been used to reveal key inputs driving the output uncertainty. Just a few works have explored sensitivity analysis in the context of imprecise probability and P-box modeling. R. Schóbi and B. Sudret [29] investigated global sensitivity analysis using sparse polynomial chaos expansion to reduce the computational cost of the analysis. S. Ferson and W. T. Tucker [30] explored a novel sensitivity analysis method by P-box 'pinching' and convolution. E. Patelli, M. Broggi, M. D. Angelis and D. A. Alvarez [28] proposed a comparison of 2 sensitivity analysis methods, 1) based on Hartley-like measure of non-specificity and 2) based on classical global sensitivity analysis by Sobol indices estimation.

In this paper, we use a generalized sensitivity analysis method, similarly to [30], which uses "pinching" to estimate the importance of an input P-box one-at-a-time. A P-box can be pinched in several ways: 1) it can be pinched to a specific CDF (i.e. no epistemic uncertainty involved); 2) it can be replaced with a precise point-point value (i.e. neither epistemic nor aleatory uncertainty involved); 3) it can be pinched to an interval with zero variance (i.e. only epistemic uncertainty involved). Independently from the adopted pinching scheme, the reduced uncertain factor must be included in the original P-box bounds. Variance-based methods use relative reduction in variances to quantify the reduction in uncertainty. Analogously, a suitable metric to quantify the reduction in P-boxes uncertainty has to be selected. The area between the lower and upper output CDFs $\delta$ is selected a representative metric and defined as follows [30]:

$$
\delta=\int_{-\infty}^{+\infty}\left|\bar{F}_{Y}(y)-\underline{F}_{Y}(y)\right| d y
$$

where $\delta$ measures the level of (epistemic) uncertainty of the output P-box. The metric $\delta$ is then used to quantify the sensitivity of the output to a reduction in uncertainty for the $i^{\text {th }}$ input, as follows:

$$
S_{i}=100 \cdot\left(\frac{\delta-\delta_{i}}{\delta}\right)
$$

where $\delta_{i}$ is the area after pinching the input variable $i$. The higher the reaction is, the higher the importance of the input uncertainty [30].

Figure 2 presents graphically the P-box pinching procedure. Two uncertain input $x_{1}$ and $x_{2}$ are modelled using a distribution-free P-box and a distributional P-box, respectively. Uncertainty is propagated to a model output $y=f(x)$ (black solid line in the bottom P-box). Then, the P-box modelling $x_{2}$ (top left P-box) is pinched to a specific CDF, which leads to a reduction in uncertainty (area) for the output P-box (dotted green line). Similarly, the P-box modelling $x_{2}$ (top right P-box) is pinched to a crisp point-value and the reduction in area quantified in the output (dotted marked red line). Accordingly to the method, the sensitivity of $y$ to the factor $x_{2}$ result the highest, i.e. it leads to the highest reduction in epistemic uncertainty.

The overall computational costs required to propagate P-box and to perform sensitivity analysis can be quite high and is mostly affected by time complexity of the min-max mapping in equations 5-6 and on the computational model $\mathcal{R}$. Develop a computationally efficient surrogate model specifically fit to replace costly high-fidelity model $\mathcal{R}$ is necessary to reduce the computational burden of these analysis and, at the same time, preserving a good output accuracy. 


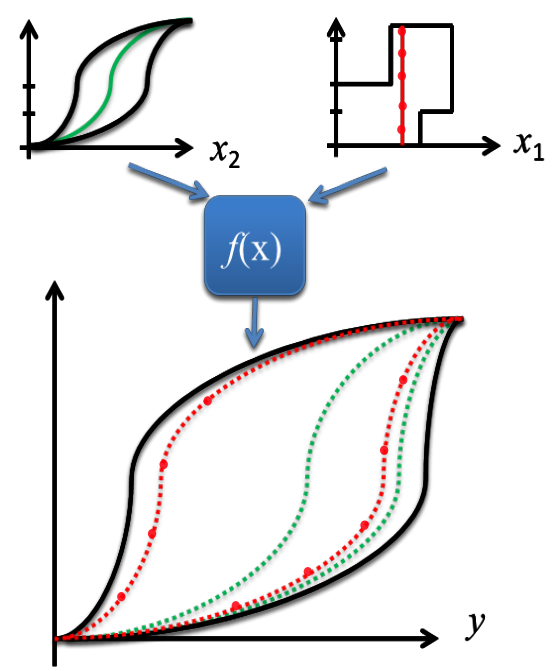

Figure 2: Example of sensitivity analysis on model $f(x)$ by pinching input P-boxes on-at-a-time. Two inputs $x_{1}$ and $x_{2}$ are pinched to crisp point-value and to a precise CDF, respectively. This induces a different reduction in area for the output P-box.

\section{Power grid probabilistic risk assessment}

Generally speaking, the risk is defined as the product of the probability of occurrence of an event (i.e. contingency) and the related consequences (i.e. severity). In order to account multiple events in an integral way, the definition is extended by summing the event's contributions as follows:

$$
R=\sum_{i=1}^{n} \mathcal{P}\left(E_{i}\right) \cdot \operatorname{Sev}\left(E_{i}\right)
$$

where $\mathcal{P}\left(E_{i}\right)$ is the probability of occurrence of the undesired event $E_{i}, \operatorname{Sev}\left(E_{i}\right)$ is the severity of the event when it occurs and $n$ is the number of events considered.

In the context of risk assessment for power grids, a risky event is an unexpected loss of one (or more) element in the system (e.g. distribution lines, transformers, generators) [8]. Each event is a so-called contingency and its severity can be measured by analyzing different factors of interest, for instance, physical quantities which are reflecting the level of stress faced by the network (e.g. a voltage drop, a frequency deviation from the nominal, excessive flow of power in the branches, etc. ). In this work, and in line with [10]-[10], the line power flows measures the overload stress in the system and its closeness to a line thermal violation. The N-1 line failures are the contingency events considered and the composite risk index $R(\zeta)$ for a given environmental-operational condition $\zeta$ is defined as:

$$
R(\zeta)=\sum_{i=1}^{N_{C}} \mathcal{P}\left(C_{i} \mid \zeta\right) \sum_{l=1}^{N_{l}} S_{O L, l}\left(C_{i}, \zeta\right)
$$


where $N_{C}$ is the total number of failures in the contingency list, $\zeta=\left\{\mathbf{L}, \mathbf{B}, l_{l}, w, \lambda_{l} ..\right\}$ is the set of structural, environmental and operational factors (e.g. load demand vector $\mathbf{L}$, wind speed $w$, line length $l_{l}$, failure rates $l a m b d a_{l}$, etc.), $C_{i}$ is the $i^{\text {th }}$ contingency listed, $S_{O L, l}$ is the overload severity for the line $l$ due to contingency $C_{i}$ in the condition $\zeta, \mathcal{P}\left(C_{i} \mid \zeta\right)$ is the probability of facing $C_{i}$ given $\zeta, N_{l}$ is the total number of lines in the system.

\subsection{A probabilistic model for weather-induced failures}

In this work, high wind intensity can induce failures in the power grid by increasing the occurrence probability of lines tripping. Thus, the grid operational state is affected by 'normal weather' and 'severe weather' as in [31]. A model for normal weather conditions describes weather-independent events, e.g. aging, maintenance, targeted attacks or manufacturing errors in general. A severe weather model captures failure events which are induced by severe weather conditions (e.g. wind-induced fatigue, structural failure of the lines due to heavy snow, etc.). Windy weather is considered here and, for simplicity but without loss of generality, assumed uniform over the whole system. A wind speed prediction is considered available (e.g. by weather forecast) but uncertainty is affecting its expected intensity. An error affecting the prediction for the wind speed intensity $w$ is assumed normally distributed with probability density function:

$$
f_{W}(w)==\frac{1}{\sqrt{2 \pi \sigma_{w}}} e^{-\frac{\left(w-\mu_{w}\right)^{2}}{2 \sigma_{w}^{2}}}
$$

where $\mu_{w}$ and $\sigma_{w}$ the mean and standard deviation of the prediction error.

High wind increase the grid operative risk, and in Ref. [8] it has been shown a correlation between the occurrence rate of transmission lines failures and wind intensity (e.g. friction-induced fatiguing, structure's joint failures, trees branches falling in the proximity of the line, etc). High wind speed is assumed to increase the total failure rate $\lambda_{l}$ for a transmission line $l$ as follows [31]:

$$
\lambda_{l}= \begin{cases}\lambda_{l, n o m} & \text { if } w \geq w_{\text {critic }} \\ \lambda_{l, n o m}+\lambda_{l, w} & \text { otherwise }\end{cases}
$$

where $\lambda_{l, n o m}$ is the failure rate in $\left[\frac{o c c}{\mathrm{~km} \cdot \mathrm{h}}\right]$ during normal weather conditions and $\lambda_{l, w}$ is the contribution to the line failure rate due to high wind speed [? ]:

$$
\lambda_{l, w}(w)=\lambda_{l, \text { nom }}\left(\frac{w^{2}}{w_{\text {critic }}^{2}}-1\right) \alpha_{w}
$$

were $\alpha_{w}$ is a regression parameter obtained from failure data in [] and $W_{\text {critic }}$ is the critical wind speed assumed to be $8[\mathrm{~m} / \mathrm{s}]$. The failure rate due to a wind event has a strong relation with the wind intensity, following a quadratic law. It can be observed that for wind speed less or equal to the critical wind speed ( $\left.w \leq w_{\text {critic }}\right)$ the wind contribution to the failure rate is null.

In traditional deterministic risk assessment, the impact of each failure is considered but the likelihood of facing the contingency is not. In probabilistic risk assessment, the probability of components failure is estimated based on the available information and historical data. The probability of each contingency takes into account the failure of a distribution line by a Poison distribution function as in [9]: 


$$
\mathcal{P}\left(C_{l} \mid \zeta\right)=\left(1-\exp \left(l_{l} \cdot \lambda_{l}(\zeta)\right)\right) \cdot \exp \left(-\sum_{j \neq l, j=1}^{N_{l}} \lambda_{j}(\zeta) \cdot l_{j}\right)
$$

where $\mathcal{P}\left(C_{l} \mid \zeta\right)$ is the probability of line contingency $l$ in the next $1 \mathrm{~h}, \lambda_{l}$ is the weather-dependant failure rate of the distribution line $l$ per unit time and length and $l_{l}$ source is the length of the $l^{\text {th }}$ line. The expression 15 holds if the contingencies list include all the single line failures which are considered as mutually exclusive events.

\subsection{Line overload severity model}

A over-load severity function $S_{O L, l}$ is specifically defined for each line in the power grid and it quantify the extent of a violation on the line (i.e. proximity to the line thermal limit). The line percentage of rating $P R_{l}=\frac{f_{l}}{f_{\text {emerg }, l}}$ i used to measure how close is the line flow $f_{l}$ to a deterministic failure threshold, i.e. the emergency rating $f_{\text {emerg, },}$. The $S_{O L, l}$ is defined as follows [10]:

$$
S_{O L, l}\left(P R_{l}\right)= \begin{cases}d \cdot P R_{l}+c & \text { if } P R_{l} \geq P R_{l}^{\min } \\ 0, & \text { otherwise }\end{cases}
$$

where the severity $S_{O L, l}$ is zero for $P R_{l}$ values less than a safety limit $P R_{l}^{s a f e}=0.9$. The deterministic limit for the violation of line $l$ is $P R_{l}=1$, the near violation region is $0.9 \leq P R_{l}<1$, and the value $P R_{l}$ under 0.9 is regarded as safe, $d=10$ and $c=-9$.

\subsection{Direct Current Optimal Power Flow (DC-OPF)}

The Direct Current (DC) power flow is a linear approximation of the Alternate Current (AC) power flow which accounts for just active power, neglecting power loses and reactive power management [32]. It is often used to alleviate the computational cost of numerically intensive codes and it has always a feasible solution. The DC power balance equations can be written as follows [32]:

$$
P_{k} \approx \sum_{i=1}^{N_{b}} B_{i, k} \theta_{i, k}
$$

were $N_{b}$ is the number of nodes in the network, $P_{k}$ is the active power injected in the node $k$, $\theta_{i, j}$ and $B_{i, k}$ are the voltage angle difference and the line susceptance between node $i$ and $k$, respectively. The equation 17 is obtained under the following DC power flow assumptions of flat voltage profile $\left(\left|V_{i}\right|=1\right.$ per unit. $\forall$ nodes $\left.i\right)$, small voltage angle differences $\left(\sin \left(\theta_{i, k}\right) \approx \theta_{i, k}\right)$ and negligible resistance $(R \ll X)$.

The DC power flow equations can be used to select an optimal production profile which minimizes the production costs for the system. This is commonly referred to as a DC optimal power flow (DC-OPF) defined as follows:

$$
\begin{array}{cl}
\min _{\mathbf{x}} & f(\mathbf{x}) \\
\text { s.t. } & g(\mathbf{x})=0, \\
& h(\mathbf{x}) \leq 0, \\
& \mathbf{x}_{\min } \leq \mathbf{x} \leq \mathbf{x}_{\max } . \\
& 8
\end{array}
$$


where $f$ is a cost function, $g$ is a set of equality constraints (e.g. power balance), $h$ is a set of inequality constraints (e.g. maximum voltage angles), whereas $\mathbf{x}_{\min }$ and $\mathbf{x}_{\max }$ define the lower and upper bounds on the power profile $\mathbf{x}$ (i.e. generators capacity), respectively.

\subsection{Line Outage Distribution Factors (LODFs)}

The Power Transfer Distribution Factors (PTDFs) are linear sensitivity measures obtained under the DC assumption. The PTDFs indicate the incremental change in real power flowing on transmission lines due to a power transfer between two regions of the grid (e.g. two areas, two nodes, etc). The matrix of Line Outage Distribution Factors (LODFs), can be directly derived from the PTDFs and it quantifies the ratio of change in line active flows conditional to the removal of transmission lines one-at-a-time. Specifically, each one of its elements $d_{l}^{k}$ quantify the impact of removing a transmission line $k$ on the active flow in the line $l$, see [33]-[34]-[35] for further details. Thus, provided a pre-outage active flow in the line $l\left(f_{l}\right)$, it is possible to compute directly the post-contingency flow after failure of the line $k\left(f_{l}^{k}\right)$ as follows [11]:

$$
f_{l}^{k}=f_{l}+d_{l}^{k} f_{k}
$$

were $d_{l}^{k}$ is the element on the $l^{\text {th }}$ row and $k^{\text {th }}$ column of the LODF matrix.

In this sense, LODFs can be exploited as a power flow surrogate to approximate post-contingency flows, line percentages of rating and, finally, overload severity scores. The total overload risk faced by the system (see Eq.11) can be obtained with a single run of the pre-continency power flow, with no need to solve additional power flow equations in post-contingency scenarios.

\section{The proposed framework based on the post-contingency power-flow surrogate}

A computationally cheap risk assessment framework is proposed by exploiting the approximation capability of the Line Outage Distribution Factors. Algorithm 1 summarizes the procedure for the surrogate risk assessment model proposed in this paper. First, a prediction for the future environmental-operational conditions (e.g. a forecast) and the grid data are provided in the vector $\zeta$. Then, a pre-contingency DC optimal power flow is computed to estimate the line active flows $f_{k}$ in normal operational conditions, PTDFs and LODFs are computed. After the pre-contingency power flow evaluation, contingency analysis starts by updating the line failure rates accordingly to the expected wind speed (as described in sections 3.3), probability of failure computed as in equation 15 and post-contingency flows are directly obtained by using the Line-Outage Distribution-Factors $d_{l}^{k}$ for the N-1 line failures as in equation 18. Finally, overload severity for each line and each contingency is obtained and composite overload risk index computed as in equations 16 and 11, respectively.

The efficient risk assessment model is embedded within the generalized framework for uncertainty quantification as presented in Section 2. The framework can be utilized to perform classical uncertainty quantification tasks (e.g. Monte Carlo aleatory uncertainty propagation, correlation analysis, sensitivity analysis, etc.) or generalized uncertainty quantification tasks. For instance, the slicing algorithm can be used to evaluate the effect on the overall risk index $R(\zeta)$ of the imprecision affecting in the input parameters $\zeta$. The procedure for P-boxes propagation using the slicing method is summarized in the Algorithm 2 and Section 2.2.1 which works as follows: 


\section{Initialization:}

Input the number of $\alpha$ slices $\left(N_{\alpha}\right)$, GA starting population ( $p o p$ ), maximum number of generations (epoch) and number of imprecise input variables $N_{i}$.

\section{Slicing:}

1. Extract randomly $\alpha=\left(\alpha_{1}, \alpha_{2}, \ldots, \alpha_{N_{i}}\right)$ where $\alpha_{i} \sim U(0,1), i=1 . ., N_{i}$. Invert P-boxes and obtain the hyper-rectangle $\mathcal{I}_{X, \alpha}$ as in equation 4.

2. Start minimization and maximization with GA. Randomize initial population pop constrained within $\mathcal{I}_{X, \alpha}$. Evaluate population fitness $R(\zeta)$, perform selection, crossover, mutation to produce offspring chromosomes. Evaluate offspring fitness function and repeat in evolutionary procedure until epoch is reached.

3. Save the resulting $[\underline{R}(\zeta), \bar{R}(\zeta)]_{\alpha}$ providing output bounds for the first $\alpha$-slice. Repeat steps 1,2 and 3 are repeated until $N_{\alpha}$ slices are evaluated.

4. The intervals $[\underline{R}(\zeta), \bar{R}(\zeta)]_{1},[\underline{R}(\zeta), \bar{R}(\zeta)]_{2}, \ldots,[\underline{R}(\zeta), \bar{R}(\zeta)]_{N_{\alpha}}$ are used to construct the risk Pbox.
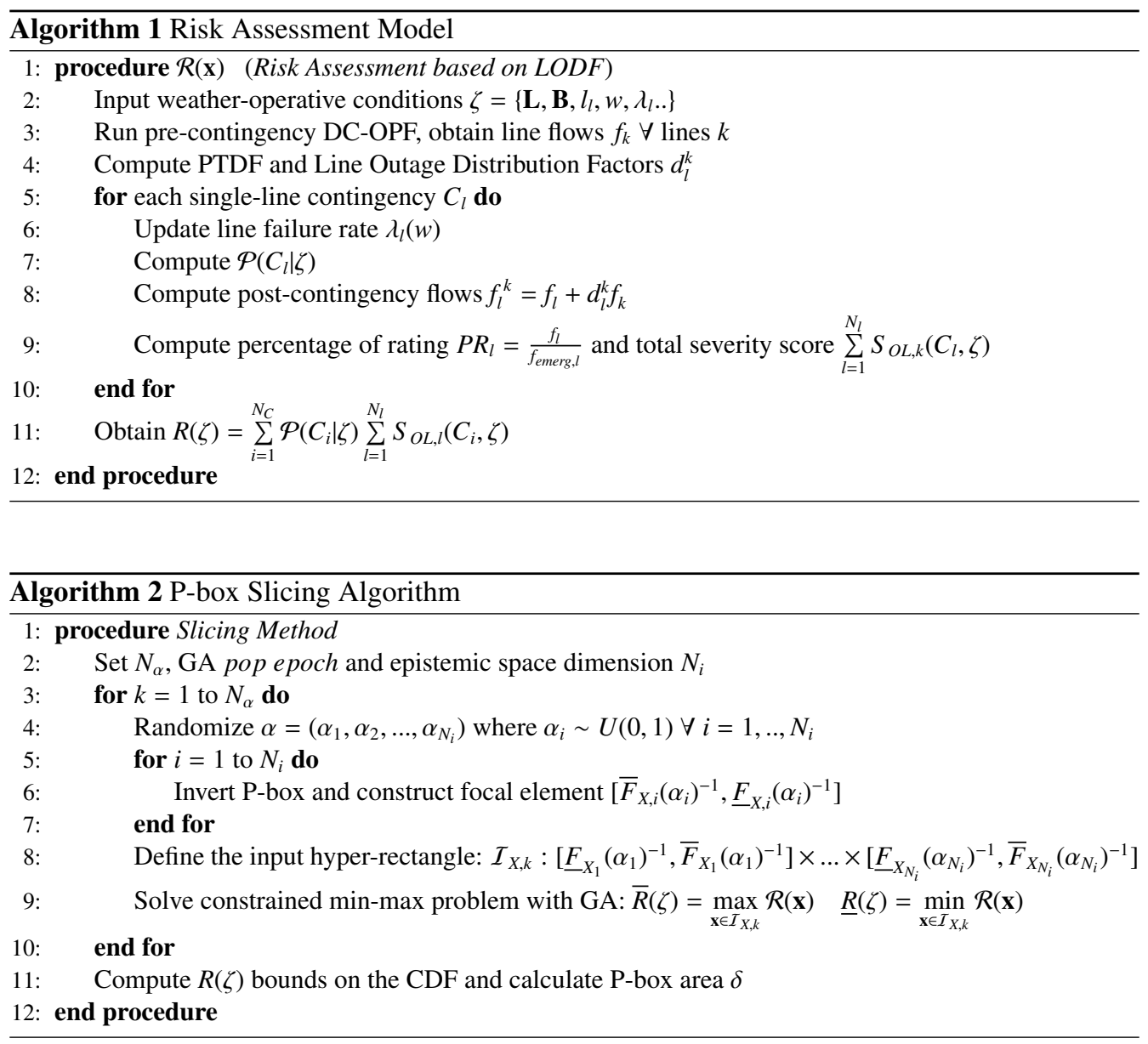


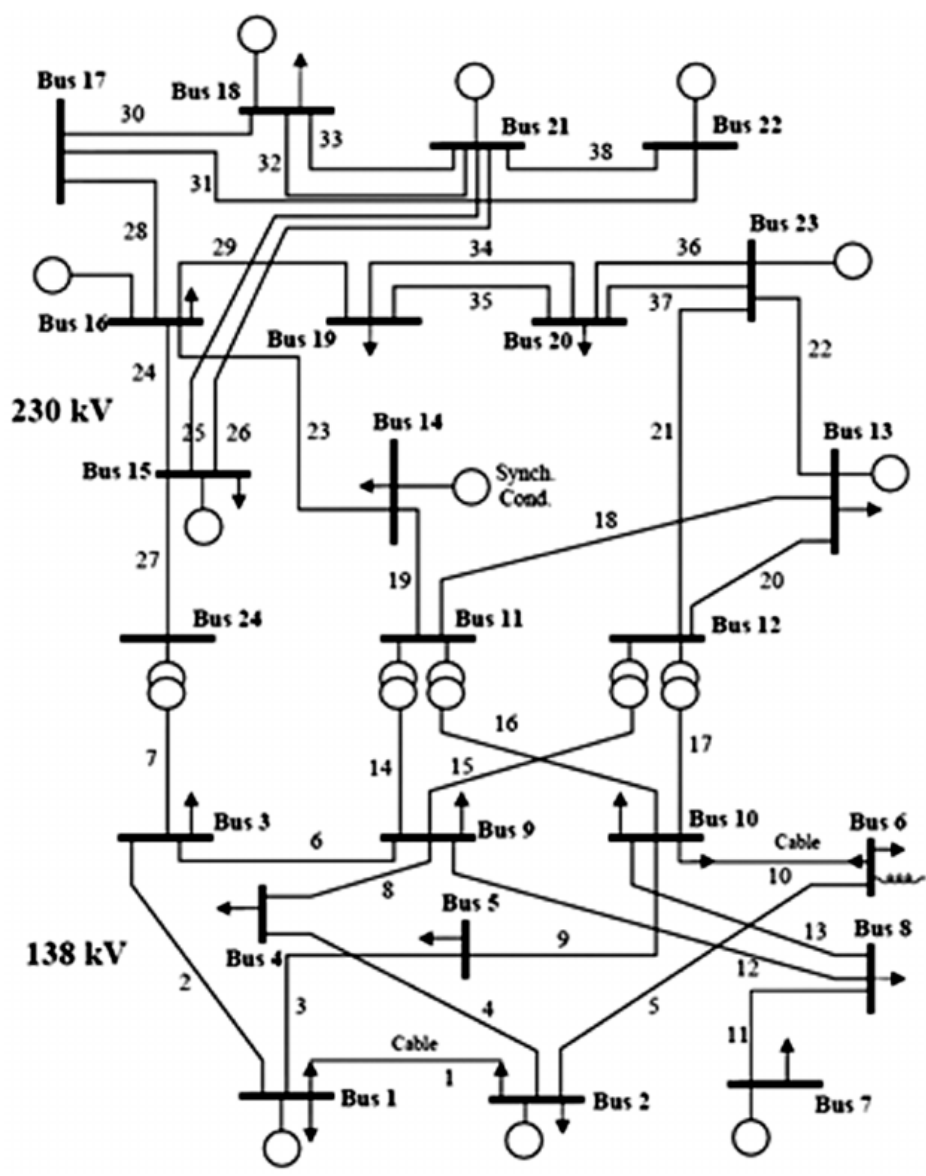

Figure 3: The IEEE 24 nodes reliability test system [36].

\section{Case Study}

A modified version of the IEEE reliability test system [7] is used to test the framework. Figure 3 depicts the network architecture, the 38 lines connecting the 24 nodes, the main generating units, the transformers and the locations of the 17 load nodes (the nodes with arrows in figure). Table 1 presents the estimated values of the line failure rates $\lambda_{l}$ expressed in $\left[\frac{\text { occurrence }}{h}\right]$. Table 2 shows the connectivity structure of the lines and corresponding identification number (ID). Additional details such as design peak loads, lines susceptances or lines thermal rating are not reported here, for sake of synthesis. The reader is reminded to [36] or [7] for a full display of the network data.

\subsection{Characterization of Uncertainty}

The considered uncertain input are the 17 load demands $\left(L_{i}\right)$ in each demand node $i$, the 38 failure rates of the lines $\lambda$ and the wind speed prediction $w$ assumed to be uniform over the gird area. For each line, the estimator of the failure rate $\lambda_{l}$ is assumed to be affected by pure epistemic 


\begin{tabular}{|l|l|l|l|l|l|}
\hline ID & $\lambda_{l}$ & ID & $\lambda_{l}$ & ID & $\lambda_{l}$ \\
\hline \hline 1 & 0.0274 & 14 & 0.0023 & 27 & 0.0468 \\
2 & 0.0582 & 15 & 0.0023 & 28 & 0.3801 \\
3 & 0.0377 & 16 & 0.0023 & 29 & 0.0388 \\
4 & 0.0445 & 17 & 0.0023 & 30 & 0.0365 \\
5 & 0.0548 & 18 & 0.0457 & 31 & 0.0616 \\
6 & 0.0434 & 19 & 0.0445 & 32 & 0.0400 \\
7 & 0.0023 & 20 & 0.0457 & 33 & 0.0400 \\
8 & 0.0411 & 21 & 0.0594 & 34 & 0.0434 \\
9 & 0.0388 & 22 & 0.0559 & 35 & 0.0434 \\
10 & 0.0377 & 23 & 0.0434 & 36 & 0.0388 \\
11 & 0.0342 & 24 & 0.3801 & 37 & 0.0388 \\
12 & 0.0502 & 25 & 0.0468 & 38 & 0.0514 \\
13 & 0.0502 & 26 & 0.0468 & - & - \\
\hline
\end{tabular}

Table 1: The lines Failure rates

\begin{tabular}{|l|l|l|l|l|l|}
\hline ID & $i-j$ & ID & $i-j$ & ID & $i-j$ \\
\hline \hline 1 & $1-2$ & 14 & $9-11$ & 27 & $15-24$ \\
2 & $1-3$ & 15 & $9-12$ & 28 & $16-17$ \\
3 & $1-5$ & 16 & $10-11$ & 29 & $16-19$ \\
4 & $2-4$ & 17 & $10-12$ & 30 & $17-18$ \\
5 & $2-6$ & 18 & $11-13$ & 31 & $17-22$ \\
6 & $3-9$ & 19 & $11-14$ & 32 & $18-21$ \\
7 & $3-24$ & 20 & $12-13$ & 33 & $18-21$ \\
8 & $4-9$ & 21 & $12-23$ & 34 & $19-20$ \\
9 & $5-10$ & 22 & $13-23$ & 35 & $19-20$ \\
10 & $6-10$ & 23 & $14-16$ & 36 & $20-23$ \\
11 & $7-8$ & 24 & $15-16$ & 37 & $20-23$ \\
12 & $8-9$ & 25 & $15-21$ & 38 & $21-22$ \\
13 & $9-10$ & 26 & $15-21$ & - & - \\
\hline
\end{tabular}

Table 2: The lines identification numbers (ID) and nodes connectity (line connecting node $i$ to node $j$ )

uncertainty. This is a typical situation for reliable components for which only a few failure data (or no data at all) is available for the statistical analysis. Failure rates are assumed to lay within an interval $\left[\underline{\lambda}_{1}, \bar{\lambda}_{l}\right]$, where the upper and lower bounds are known to be equal to $\pm 2 \%$ of the values presented in Table 1.

The loads naturally change in time (e.g. from day to night or from winter to summer time) and, commonly, a large volume of data is available to build probabilistic models, i.e. historical time series collected over several years. However, due to recent trends in the power grid sector (e.g. allocation of renewable sources, distributed generators, etc.), the reliability of probabilistic models describing the load variability is likely to decrease. Thus, the loads are considered affected by both aleatory and epistemic uncertainty (e.g. due to a lack of knowledge on the effect of new technological developments) and P-boxes used to model this uncertainty. The family of the parent distribution is assumed to be Gaussian and imprecision affect the mean and standard deviation. For each load, the mean value $\mu\left(L_{i}\right)$ of the load distribution is assumed to be $\pm 2 \%$ of a design peak load, presented in [36]. Similarly, the standard deviations are assumed to be imprecisely defined within the intervals $\left[\underline{\sigma}\left(L_{i}\right), \bar{\sigma}\left(L_{i}\right)\right]=\left[0.05 \mu\left(L_{i}\right), 0.05 \bar{\mu}\left(L_{i}\right)\right], i=1, \ldots, 17$. Finally, a prediction for the wind speed is considered and assumed to be normally distributed with $\mu_{w}=6$ and $\sigma_{w}=1$, respectively. Imprecision is assumed affecting $\mu_{w}$ which lays within the $\pm 10 \%$ error interval $[5.4,6.6]$.

\section{Results and discussion}

\subsection{Comparison between the risk surrogate and an high-fidelity AC-OPF risk model}

In order to verify the proposed risk assessment surrogate and assess its effectiveness in performing classical uncertainty quantification analysis, we compare its results against a highfidelity model. The high-fidelity risk assessment model is based on AC-OPF, as proposed in [14]. The contingency list includes all the N-1 line failures and the consequences of a line failure are assessed by solving AC-Power Flow in a contingency state [37]. Specifically for the proposed 


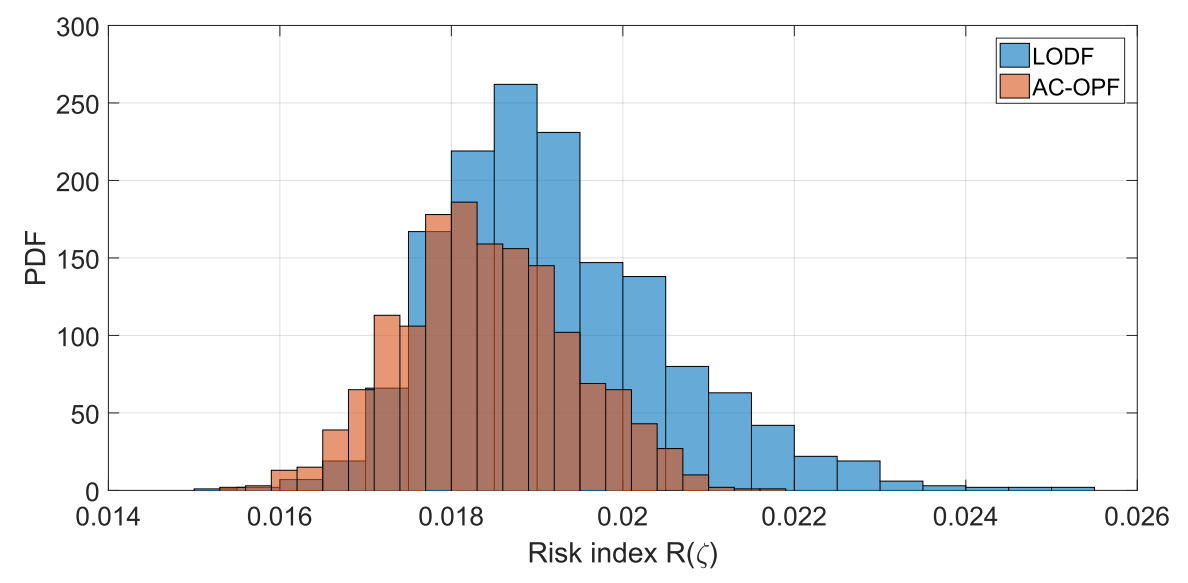

Figure 4: The $R(\zeta)$ PDFs using the proposed risk model surrogate and the AC-OPF model.

case study, a single high-fidelity risk calculation requires 1 AC-OPF (pre-contingency dispatch) and $38 \mathrm{AC}$ power flows (post-contingency consequence evaluation), for an indicative wall-clock time of 3.8 second. Although this may seems a relatively small computational cost for deterministic analysis, it can be quite intensive for UQ tasks. Computational cost which can result even prohibitive when generalized UQ method are adopted (e.g. P-box sensitivity and propagation). The proposed surrogate requires just a single DC-OPF run (pre-contingency dispatch) and postcontingency scenario are approximated by using LODF. It requires approximately 0.09 seconds to obtain $R(\zeta)$ (on a standard 2-cores, 4.0 Gb RAM, 1.7 GHz processor machine).

Monte Carlo analysis has been used to propagate the aleatory uncertainty in the loads and the wind speed. In compliance with the aims of this verification analysis, it has been assumed no epistemic uncertainty is affecting the parameters of the probabilistic model, thus performing classical UQ. Figure 4 presents the resulting PDFs, obtained by using 1500 independent samples from the aleatory uncertain variables. It can be observed that surrogate and high-fidelity models provide quite similar results. This points out a good approximation capability of the proposed surrogate. On the other hand, our surrogate slightly overestimate the upper tail of the risk distribution. Although it is conservative from a risk prospective, this can be seen as a limitation of the proposed model, which is incapable of fully capture all the relevant grid behaviors. For instance, highly non-linear conditions for which the validity of the DC assumptions slacken. Nonetheless, it provides a fairly good approximation of the $R(\zeta)$ PDF moments $(\mu=0.0192$ and $\mu=0.0014)$ when compared to the AC-OPF results $(\mu=0.0184$ and $\sigma=0.001)$. Moreover, our surrogate greatly reduces the computational cost of the UQ analysis, up to a $98 \%$ reduction. In fact, for the $1500 \mathrm{MC}$ samples, it provides the solution in about 145 seconds, substantially less than the 6200 seconds needed to solve the high-fidelity model (on a 2-cores, 4.0 Gb RAM, 1.7 $\mathrm{GHz}$ processor machine).

In order to better explore the goodness of the surrogate. correlation analysis is also proposed. Specifically, the Persons' correlation coefficients linking $R(\zeta)$ to the 17 loads have been estimated and graphically presented in Figure 5. It can be observed a good agreement between 

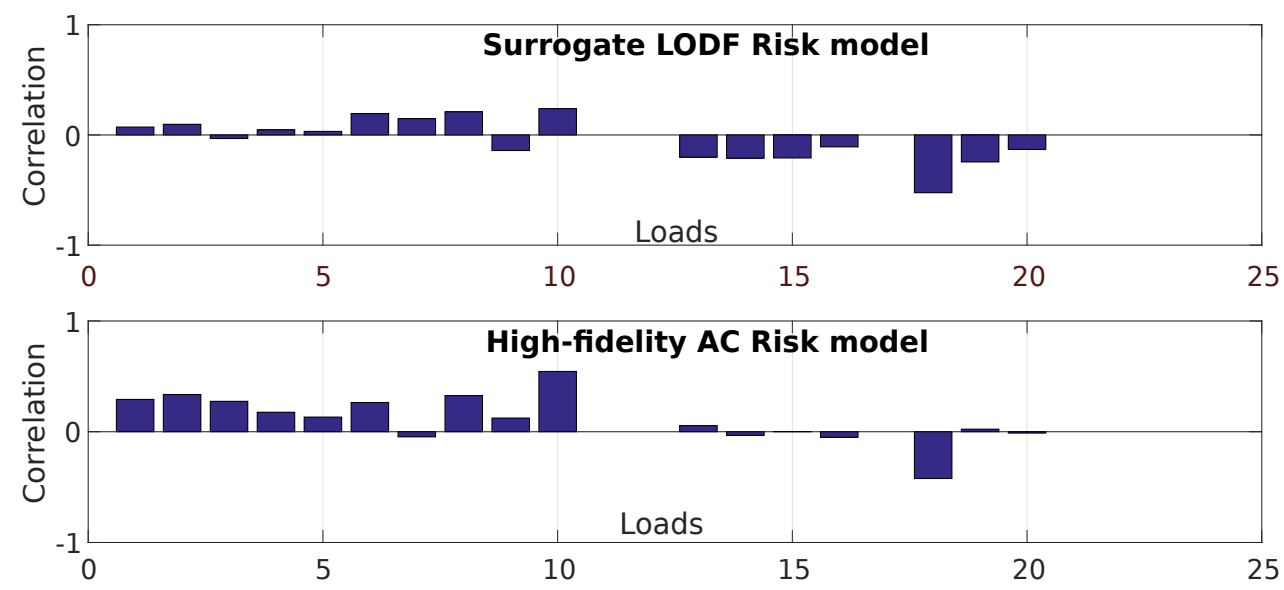

Figure 5: Persons' correlation coefficients between $R(\zeta)$ and loads for the proposed risk surrogate and the AC-OPF model.

the input-output correlations of the two models. Both identify a positive correlation between the overload risk and the loads in the southern part of the grid, i.e. nodes from 1-10 (with a little discrepancy in node 9). A negative correlation has been observed with the load in node 18, which indicates a reduction in the overload in post-contingency risk for increasing demand in node 18. This result is in good agreement with previous analysis [38] and can be explained by looking at the generators production profile. Generators in the upper part of the grid (e.g. generators in nodes $13,15,18)$ are associated with lower generation costs. This lead to the maximum exploitation of their production capacity, independently from the load profile realization. Consequently, when electrical power is demanded (and consumed) locally, less power will be flowing from the northern area to the southern area of the network, thus reducing the risk of facing more severe post-contingency overflow scenarios.

\subsection{Imprecise probabilistic analysis}

After verification of the proposed model in performing classical UQ analysis, the risk surrogate model has been adopted to perform a generalized uncertainty analysis on the system, i.e. accounting for both sources of aleatory and epistemic uncertainty and discerning between the effect of the two. Probability boxes have been used to characterize the uncertainty affecting loads, wind speed and failure rate as described in...

\subsubsection{Slicing method}

The slicing algorithm 2 has been used to propagate P-box uncertainty to the risk index. Two independent slicing propagation analyses have been performed adopting 20 and 100 slices respectively. Genetic algorithm optimizer has been adopted as described in [11] to approximate the minimum and maximum risk in each sampled hyper-rectangle. The Genetic Algorithm parameters have been tuned empirically. It has been observed that for a starting population of just 10 individuals and for about 100 iterations the risk index results close to convergence. Hence, pop $=10$ and epoch $=100$ have been selected for the risk minimization and maximization routine. 


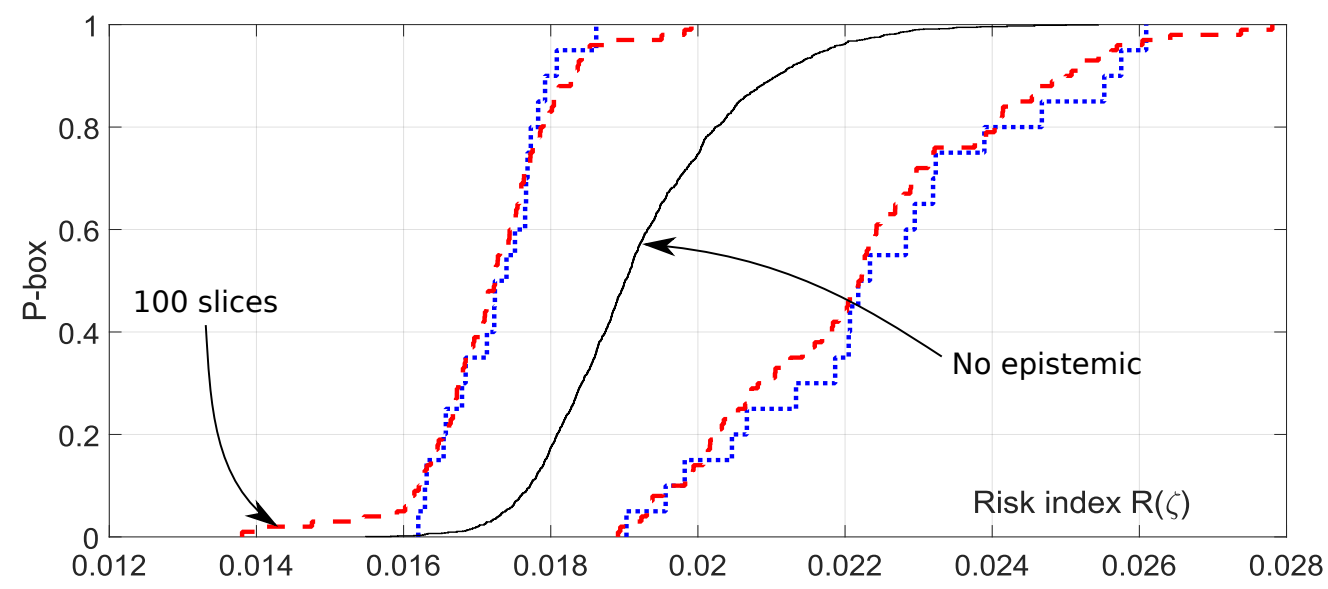

Figure 6: Comparison between the CDF obtained considering only aleatory uncertainty (black solid line) and P-boxes obtained for 20 slices (blue dotted lines) and 100 slices (red dashed lines).

Figure 6 shows the generalized uncertainty quantification results for the overload risk index. Two P-boxes have been displayed, resulting from $N_{\alpha}=20$ (blue dotted lines) and $N_{\alpha}=100$ slices (red dashed lines), respectively. AS expected, the bounds obtained using the generalized uncertainty method include the classical result (black solid line CDF) and, when compared, generalized UQ shows a possible underestimation of the risk if imprecision is not accounted for. To clarify the idea consider a case where the system operator decides for a maximum allowed overload risk of 0.023. Adopting a classical UQ approach, the probability of meeting this requirement is approximately 0.99 (i.e. due to inherent variability in the environmental-operational conditions, $1 \%$ of the possible scenarios have an unacceptable risk). Conversely, accounting for imprecision, the likelihood of fulfilling the requirement can be anywhere between 1 and about 0.7. This means that between $30 \%$ and $0 \%$ of possible scenarios do not comply with the risk requirement (due to epistemic uncertainty). Thus for this example, the worst case scenario for classical UQ results (1\%) strongly underestimate the true worst-case scenario obtained by generalized UQ $(30 \%)$.

The risk P-box area from $N_{\alpha}=100$ slices is estimated to be $\delta=0.0057$. To investigate further this results, 10 independent slicing algorithm have been run with the same setup and error on the $\delta$ results analysed. The average error in area resulted approximately $4.7 \%$ and it is likely due to an early convergence of the GA optimization (truncated at 100 epochs). Nonetheless, this error margin was considered acceptably low and the estimate precise enough to carry on with sensitivity analysis.

\subsubsection{Sensitivity Analysis}

Sensitivity analysis on the epistemic space has been performed by pinching each load $L_{i}$ to the mean empirical CDF (average between upper and lower P-box bounds). Similarly, line failure rates have been pinched to a reduced interval (i.e. an improved estimator, from the initial $\pm 2 \%$ to a $\pm 1 \%$ of the estimated $\lambda_{l}$ ). The reduced epistemic space has been propagated through the risk model by the slicing method (Algorithm2) and setting $N_{\alpha}=50, N_{i}=55$ (i.e. $17 L_{i}$ and 38 
$\left.\lambda_{l}\right)$ and GA pop $=10$ and $e p o c h=50$.

It is worth remarking that P-box sensitivity can be very intensive computationally speaking, i.e. the computational complexity is equal to $N_{i}$ times the already high cost of the slicing methods. Thus, a high-performance computing strategy has been designed to forward each $\alpha$-slice to a core of a 8-cores computers cluster. Thanks to the efficient risk assessment framework, Algorithm 1, the overall computational time for the analysis was about 13.3 hours. The same analysis would have been infeasible using the high-fidelity model (indicatively 20 days). Figure 7 shows the effect on the $R(\zeta)$ P-box bounds when pinching the loads and Table 3 displays ranking results based on their sensitivity score $S_{i}$ (i.e. P-box area reduction as in equation 9).

Table 3: Ranks of the load imprecision with respect to $S_{i}$.

\begin{tabular}{llllll}
\hline Load ID & $S_{i}$ & rank & Load ID & $S_{i}$ & rank \\
\hline$L_{18}$ & $25.8 \%$ & 1 & $L_{16}$ & $8.5 \%$ & 10 \\
$L_{19}$ & $20.2 \%$ & 2 & $L_{5}$ & $7.9 \%$ & 11 \\
$L_{3}$ & $13.9 \%$ & 3 & $L_{7}$ & $7.1 \%$ & 12 \\
$L_{14}$ & $13.6 \%$ & 4 & $L_{10}$ & $6.4 \%$ & 13 \\
$L_{15}$ & $11.8 \%$ & 5 & $L_{4}$ & $6.1 \%$ & 14 \\
$L_{20}$ & $11.7 \%$ & 6 & $L_{6}$ & $6 \%$ & 15 \\
$L_{13}$ & $10.8 \%$ & 7 & $L_{9}$ & $5 \%$ & 16 \\
$L_{1}$ & $8.6 \%$ & 8 & $L_{8}$ & $1 \%$ & 17 \\
$L_{2}$ & $8.6 \%$ & 9 & & & \\
\hline
\end{tabular}
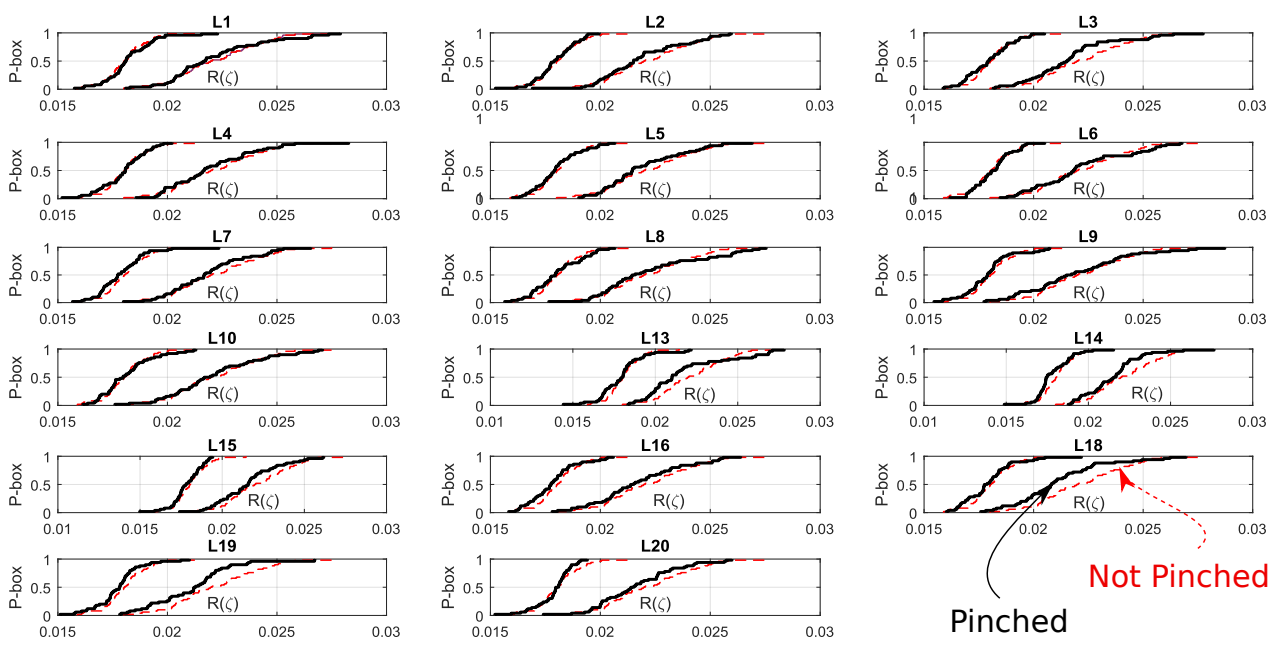

Pinched

Figure 7: Risk probability bounds before and after pinching the 17 loads. For each $\alpha$ level, the input load is pinched to the mean between upper and lower bounds.

The sensitivity analysis points out that the uncertainty affecting load in node 18 has the largest contribution to the risk of epistemic uncertainty. This is in accordance with previous 
analysis [11]. Similarly, the epistemic uncertainty affecting the loads at node 19, 3 and 14 has also a sensible effect on the risk imprecision. By reducing the uncertainty in the load 18 (i.e. by pinching) an area reduction of $25.8 \%$ has been observed for the risk P-box. Pinching the loads 19 , 3 and 14 lead to a $20.2 \%, 13.9 \%$ and $13.5 \%$ reduction, respectively. This is a piece of important information which is exploitable for future collection of data or uncertainty reduction schemes. For instance, it can be advised: 1) A better monitoring of the load 18 and 19;2) a dedicated control policy leading to a reduction in the load uncertainty in 18 (e.g. by peak shaving or by load curtailment ). For what concerns the other imprecise factors considered in this analysis (the other loads and the failure rates), their contributions to the reduction in the area seems to be less relevant, i.e. a reduction between $1 \%$ and $10 \%$, and are not reported here.

\section{Conclusion}

In this paper, a power grid probabilistic risk assessment framework has been introduced and a surrogated model proposed for generalized uncertainty quantification. The surrogate model is used to speed up time-costly risk calculations and is embedded within a generalized framework for aleatory-epistemic uncertainty propagation and sensitivity analysis. Its accuracy and computational cost are tested against a high-fidelity risk model based on the AC power flow method and accounting for the N-1 single line failures. The surrogate model results are comparable in accuracy but with a great reduction in the computational cost of the analysis, up to a $97 \%$ reduction. Probability boxes are used to characterize the uncertainty in the gird power loads, environmental factors, and components availability. The Slicing method and sensitivity by pinching are proposed to analyze the effect of mixed aleatory-epistemic uncertainties on the output of the surrogate model. Two of the loads have been identified as key epistemic uncertain factors which are preventing the most from a crisp calculation of the system risk score. The proposed framework is a valuable tool which enables the examiners to efficiently (thanks to the surrogate model) analyze the extent of reducible and irreducible uncertainty on the grid risk. Furthermore, the framework can be used to identify key sources of lack of information and inherent variability to be identified. This is valuable information which can be used to 1) design smarter data collection schemes, 2) perform uncertainty reduction to provide less uncertain risk score, 3 ) Jdesign enhanced online risk assessment models and design better operational policies with greater awareness of associated risks.

\section{References}

[1] Q. Chen, J. D. McCalley, Identifying high risk n-k contingencies for online security assessment, IEEE Transactions on Power Systems 20 (2005) 823-834.

[2] D. Gan, R. J. Thomas, R. D. Zimmerman, Stability-constrained optimal power flow, IEEE Transactions on Power Systems 15 (2000) 535-540.

[3] N. Amjady, H. Sharifzadeh, Security constrained optimal power flow considering detailed generator model by a new robust differential evolution algorithm, Electric Power Systems Research 81 (2011) 740 - 749.

[4] H. Harsan, N. Hadjsaid, P. Pruvot, Cyclic security analysis for security constrained optimal power flow, IEEE Transactions on Power Systems 12 (1997) 948-953.

[5] Z.-L. Gaing, R.-F. Chang, 'security-constrained optimal power flow by mixedinteger', Proc. IEEE Power Engineering Society General Meeting (2006) 1-2. Cited By 2.

[6] J. McCalley, S. Asgarpoor, L. Bertling, R. Billinion, H. Chao, J. Chen, J. Endrenyi, R. Fletcher, A. Ford, C. Grigg, G. Hamoud, D. Logan, A. Meliopoulos, M. Ni, N. Rau, L. Salvaderi, M. Schilling, Y. Schlumberger, A. Schneider, C. Singh, Probabilistic security assessment for power system operations, in: Power Engineering Society General Meeting, 2004. IEEE, pp. 212-220 Vol.1. 
[7] F. Xiao, J. McCalley, Power system risk assessment and control in a multiobjective framework, Power Systems, IEEE Transactions on 24 (2009) 78-85.

[8] J. McCalley, F. Xiao, Y. Jiang, Q. Chen, Computation of contingency probabilities for electric transmission decision problems, in: Intelligent Systems Application to Power Systems, 2005. Proceedings of the 13th International Conference on, pp. 540-545.

[9] M. Ni, J. McCalley, V. Vittal, T. Tayyib, Online risk-based security assessment, IEEE Transactions on Power Systems 18 (2003) 258-265. Cited By 243.

[10] R. Rocchetta, Y. Li, E. Zio, Risk assessment and risk-cost optimization of distributed power generation systems considering extreme weather conditions, Reliability Engineering \& System Safety 136 (2015) 47 - 61.

[11] R. Rocchetta, E. Patelli, Imprecise probabilistic framework for power grids risk assessment and sensitivity analysis, in: Risk, Reliability and Safety: Innovating Theory and Practice, pp. 2789-2796.

[12] S. Ferson, V. Kreinovich, L. Ginzburg, D. S. Myers, K. Sentz, Constructing probability boxes and Dempster-Shafer structures, volume 835, Sandia National Laboratories, 2002.

[13] R. Rocchetta, M. Broggi, E. Patelli, Do we have enough data? robust reliability via uncertainty quantification, Applied Mathematical Modelling 54 (2018) 710 - 721.

[14] R. Rocchetta, E. Patelli, Assessment of power grid vulnerabilities accounting for stochastic loads and model imprecision, International Journal of Electrical Power \& Energy Systems 98 (2018) 219 - 232.

[15] R. Zhong, Z. Zong, J. Niu, Q. Liu, P. Zheng, A multiscale finite element model validation method of composite cable-stayed bridge based on probability box theory, Journal of Sound and Vibration 370 (2016) $111-131$.

[16] R. Schőbi, B. Sudret, Structural reliability analysis for p-boxes using multi-level meta-models, Probabilistic Engineering Mechanics 48 (2017) 27 - 38.

[17] M. Beer, S. Ferson, V. Kreinovich, Imprecise probabilities in engineering analyses, Mechanical Systems and Signal Processing 37 (2013) 4-29.

[18] E. Patelli, M. Broggi, M. Angelis, M. Beer, Opencossan: An efficient open tool for dealing with epistemic and aleatory uncertainties, in: Vulnerability, Uncertainty, and Risk, American Society of Civil Engineers, 2014, pp. 2564-2573-.

[19] G. Shafer, A Mathematical Theory of Evidence, Princeton University Press, Princeton, 1976.

[20] Y. Ben-Haim, Uncertainty, probability and information-gaps, Rel. Eng. \& Sys. Safety 85 (2004) 249-266.

[21] L. Utkin, S. Destercke, Computing expectations with continuous p-boxes: Univariate case, International Journal of Approximate Reasoning 50 (2009) 778 - 798.

[22] C. Simon, F. Bicking, Hybrid computation of uncertainty in reliability analysis with p-box and evidential networks, Reliability Engineering \& System Safety 167 (2017) 629 - 638. Special Section: Applications of Probabilistic Graphical Models in Dependability, Diagnosis and Prognosis.

[23] T. Ali, H. Boruah, P. Dutta, Modeling uncertainty in risk assessment using double monte carlo method, International Journal of Engineering and Innovative Technology 1 (2012) 114-118.

[24] H. Zhang, Interval importance sampling method for finite element-based structural reliability assessment under parameter uncertainties, Structural Safety 38 (2012) $1-10$.

[25] J. Sadeghi, M. De Angelis, E. Patelli, Analytic imprecise-probabilistic structural reliability analysis, 2018.

[26] Z. Qiu, Y. Xia, J. Yang, The static displacement and the stress analysis of structures with bounded uncertainties using the vertex solution theorem, Computer Methods in Applied Mechanics and Engineering 196 (2007) 49654984. Cited By 56.

[27] R. C. Williamson, T. Downs, Probabilistic arithmetic. i. numerical methods for calculating convolutions and dependency bounds, International Journal of Approximate Reasoning 4 (1990) 89 - 158.

[28] E. Patelli, D. A. Alvarez, M. Broggi, M. de Angelis, An integrated and efficient numerical framework for uncertainty quantification: application to the nasa langley multidisciplinary uncertainty quantification challenge, 16th AIAA Non-Deterministic Approaches Conference (SciTech 2014) (2014) 2014-1501.

[29] R. Schőbi, B. Sudret, Global sensitivity analysis in the context of imprecise probabilities (p-boxes) using sparse polynomial chaos expansions, Reliability Engineering \& System Safety (2018).

[30] S. Ferson, W. T. Tucker, Sensitivity analysis using probability bounding, Reliability Engineering \& System Safety 91 (2006) 1435 - 1442. The Fourth International Conference on Sensitivity Analysis of Model Output (SAMO 2004).

[31] R. Rocchetta, E. Zio, E. Patelli, A power-flow emulator approach for resilience assessment of repairable power grids subject to weather-induced failures and data deficiency, Applied Energy 210 (2018) 339 - 350.

[32] D. Van Hertem, J. Verboomen, K. Purchala, R. Belmans, W. Kling, Usefulness of dc power flow for active power flow analysis with flow controlling devices, in: AC and DC Power Transmission, 2006. ACDC 2006. The 8th IEE International Conference on, pp. 58-62.

[33] Y.-C. Chang, W.-T. Yang, C.-C. Liu, Improvements on the line outage distribution factor for power system security analysis, Electric Power Systems Research 26 (1993) 231 - 236.

[34] J. Guo, Y. Fu, Z. Li, M. Shahidehpour, Direct calculation of line outage distribution factors, IEEE Transactions on 
Power Systems 24 (2009) 1633-1634.

[35] G. Chen, Y. Dai, Z. Xu, Z. Dong, Y. Xue, A flexible framework of line power flow estimation for high-order contingency analysis, International Journal of Electrical Power \& Energy Systems 70 (2015) $1-8$.

[36] P. Wong, P. Albrecht, R. Allan, R. Billinton, Q. Chen, C. Fong, S. Haddad, W. Li, R. Mukerji, D. Patton, A. Schneider, M. Shahidehpour, C. Singh, The ieee reliability test system-1996. a report prepared by the reliability test system task force of the application of probability methods subcommittee, Power Systems, IEEE Transactions on 14 (1999) 1010-1020.

[37] R. Rocchetta, E. Patelli, Stochastic analysis and reliability-cost optimization of distributed generators and air source heat pumps, in: 2017 2nd International Conference on System Reliability and Safety (ICSRS), pp. 31-35.

[38] R. Rocchetta, E. Patelli, B. Li, G. Sansavini, Effect of load-generation variability on power grid cascading failures, in: European Safety and Reliability Conference (ESREL 2018). 